\title{
VARIABLE STEP SIZE OF LMS ALGORITHEM USING PARTICAL SWARM OPTIMIZATION
}

\author{
Ajjaiah H.B.M ${ }^{1}$, Prabhakar V. Hunagund ${ }^{2}$ \\ ${ }^{1}$ PhD Scholars, Dept. of PG Studies and Research in Applied Electronics, Gulbarga University, Karnataka, India \\ ${ }^{2}$ Professors, Dept. of PG Studies and Research in Applied Electronics, Gulbarga University, Karnataka, India
}

\begin{abstract}
In this paper a novel method using both Particle Swarm Optimization (PSO) and least mean Square algorithm (LMS) is proposed. The main parameters tap-length and tap-weight are updated using the PSO and the LMS algorithm respectively according to the value of mean square error (MSE).By utilizing such an approach, both a fast convergence rate and a small steady-state MSE can be obtained. Although many LMS algorithmic methods perform well under certain conditions, performance can be degrade by noise and having performance sensitivity over parameter setting. In this paper, a new concept is introduced to vary the step size based upon evolutionary programming (SSLMSEV) algorithm is described. It has shown that the performance generated by this method is robust and does not require any pre-setting of involved parameters in solution based upon statistical characteristics of signal.
\end{abstract}

Keywords: KEYWORDS: PSO, tap-length, tap-weight, LMS, SSLMSEV.

\section{INTRODUCTION}

Adaptive filters are more useful in practical cases because it can adjust the system automatically with respect to timevarying environment. Two things are to be considered if for more than one objective system such as: (a) errors in optimal cases and (b) the convergence speed. Due to over ages both tap-length and weight get changed overtime and these are the key parameters to control, and hence it is required to be updated over times. So, the duo adaptation procedure is proposed in this paper. In the proposed algorithm tap-length and tap-weights are adapted using the EP and the LMS respectively, according to the MSE of corresponding taplengths and tap-weights of the linear FIR filter. The LMS based adaptation procedures and its probable demerits are discussed and thereafter the duo adaptation technique is used for simulation. Various LMS style algorithms have been introduced to update tap-weights, tap-length, and step-size. The LMS based algorithm is more famous. Because of its simplicity and robustness with moderate convergence rate towards optimal solutions whereas RLS is complex enough but has good convergence rate. The conventional LMS follows the following equation to update tap-weight vector.

$$
W_{\mathrm{k}}=W_{\mathrm{k}-1}+2 \mu \theta_{\mathrm{k}-1} x_{\mathrm{k}-1}
$$

$\mathrm{W}_{\mathrm{k}}=$ Tap - Weight vector in $\mathrm{K}^{\text {th }}$ iteration, $\mu=$ Step-size, $\mathrm{e}_{\mathrm{k}}=$ error in $\mathrm{k}^{\text {th }}$ iteration and $\mathrm{Xk}=$ Input vector.

The main drawback of the LMS algorithm is that it is sensitive to the scaling of its input $u(n)$. This makes it very hard to choose a learning rate $\mu$ that guarantees stability of the algorithm. Although LMS restrict itself in many applications. The essence of LMS algorithm is to update the adaptive filter coefficients recursively along the negative gradient of estimate error surface as shown in Fig-1. Conventional algorithm uses a fixed step-size to perform the iteration, and to get a compromise between the conflict of fast convergence and small steady-state MSE.A small step-size could ensure small MSE with a slow convergence, where as a large step-size will provide a faster convergence and better tracking capabilities at the cost of higher steady-state MSE. Therefore, fixed stepsize. LMS algorithm definitely cannot settle this contradiction. Consequently, many variable step-size algorithms were proposed to solve the problem. Though these algorithms could accelerate convergence and deduce steady-state MSE to some extent. For convergence rate, variable step-size LMS (VSLMS) and Variable length LMS (VLLMS) have been proposed [3], where step-size and tap-length are updated after a predetermined time constant. Here step-size is updated using the following equation. 


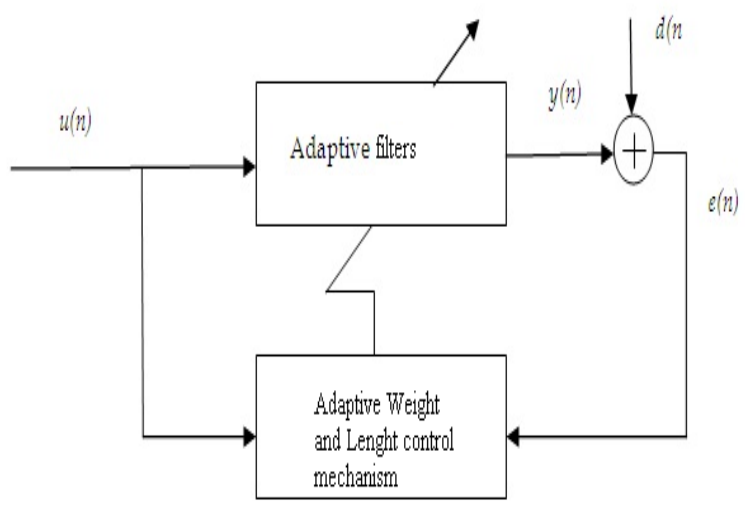

Fig-1 Block diagram of adaptive structure.

\section{RELATED WORK}

In this work we introduced a novel method to obtain an optimal step-size and an algorithm for LMS. The algorithm runs iteratively and convergence to the equalizer coefficients by finding the optimal step-size which minimizes the steadystate error rate at each iteration. No initialization for the stepsize value is required. Efficiency of the proposed algorithm is shown by making a performance comparison between some of the other LMS based algorithms and optimal step-size LMS algorithm [1] .A variations of gradient adaptive step-size LMS algorithms are presented. They propose a simplification to a class of the studied algorithms [2]. Adaption in the variable step size LMS proposed by [3] based on weighting coefficients bias/variance trade off. Authors in [4] examine the stability of VSLMS with uncorrelated stationary Gaussian data. Most VSLMS described in the literature use a datadependent step-size, where the step-size either depends on the data before the current time (prior step-size rule) or through the current time (posterior step size rule).It has often been assumed that VSLMS algorithms are stable (in the sense of mean square bounded weights), provided that the step-size is constrained to lie within the corresponding stability region for the LMS algorithm.

The analysis of these VSLMS algorithms in the literature typically proceeds in two steps [5], [6], [7].Block diagram of the basic adaptive structure which is FIR in nature, is shown in Fig. 1. Here depending on the error signal adaptive algorithm controls the tap-length and tap-weights as well and determine the fittest one. This tap-length is updated using PSO style algorithm and for every individual tap-length, tap-weights are updated using LMS algorithm. Generally both these parameters of a system are affected in a time varying environment. The proposed technique updates both parameters efficiently. In conventional PSO, crossover and mutation are performed among fittest parent and new population always replace current population. Typical values of PSO parameters are: population size $=50$, crossover rate $=0.9$, mutation rate $=0.05$. But in the proposed technique the conventional
PSO is modified as shown in the flow chart chart 1 . To achieve better convergence and therefore named as PSO style.

The flow chart of PSO style algorithm for tap-length adaptation is shown in chart 1.Here initially a pool of taplengths which are integers within a specific range is taken randomly. From the pool of tap-lengths two are taken randomly and encoded them into binary strings in representation phase. As the length of every decimal number in binary is not same, zero-padding is done to obtain equal length. First two point crossovers are performed in which the point is randomly determined. Mutation with probability 0.25 is then executed where the points are also randomly chosen. Crossover and mutation operation on the parent pair produce two offspring. Crossover does exchange the information of parent strings while mutation introduces randomness to obtain diversity in the offspring. Two child tap-lengths are then added to the parent pool. All the tap-lengths including the offspring are sorted in ascending order of their MSE. Last two tap-lengths are discarded as they are not fit and thereby population of tap-lengths is same throughout the generations. In every generation, it keeps the record of the best suited taplength having low MSE. After production of predetermined number of generation, the execution of algorithm is stopped. Weights are updated here using the conventional LMS algorithm as per equation (1).

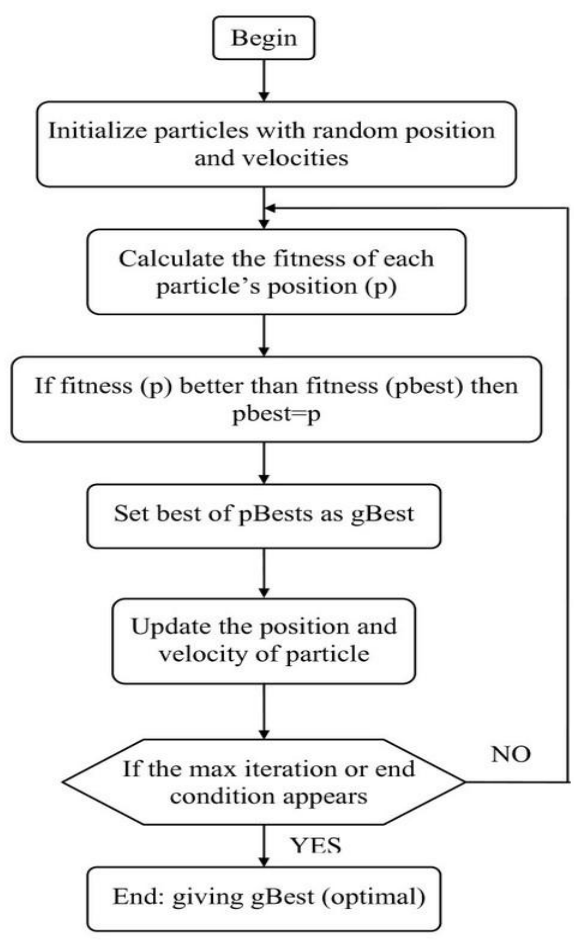

Chart -1: PSO algorithm for tap-length adaptation. 
Table 1: The pseudo code for distributed PSO (DPSO) algorithm

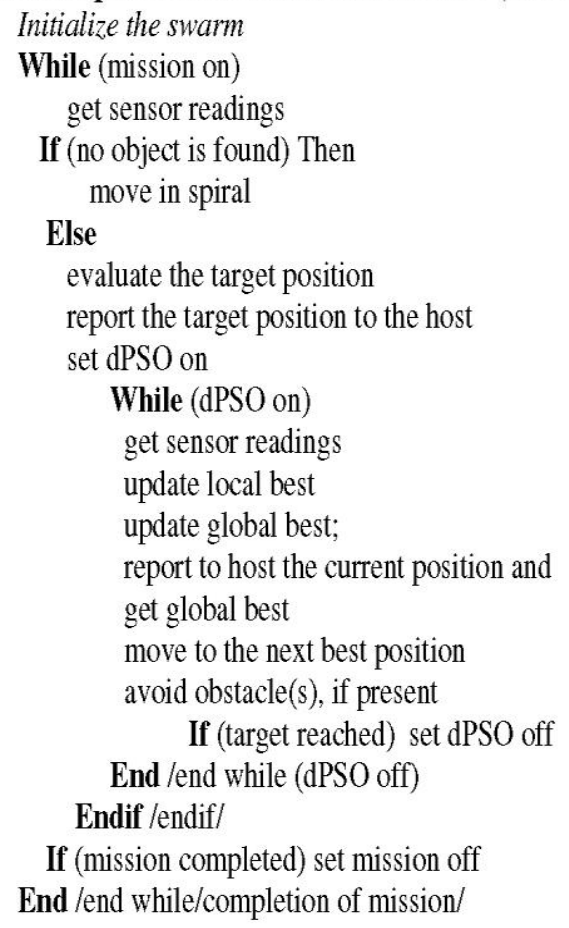

\section{SIMULATION SETUP AND DISCUSSIONS}

From the tap-length adaptation point of view, after several generations, the optimal tap-length is achieved and it gets the constant value. The performance of the proposed technique is validated by considering the unknown system function, $h=[1$

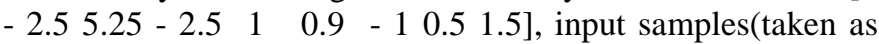
random vector $)=800$, generations $=100$. From each of these generations, one tap-length having least MSE is recorded. After hundred generations, hundred best suited tap-lengths have been plotted with corresponding MSE. While tap-length getting updated tap-weights also get updated by the LMS algorithm with step-size, $\mu=0.005$ and for every individual tap-length the MSE is estimated taking the mean of MSE from last five hundred samples.

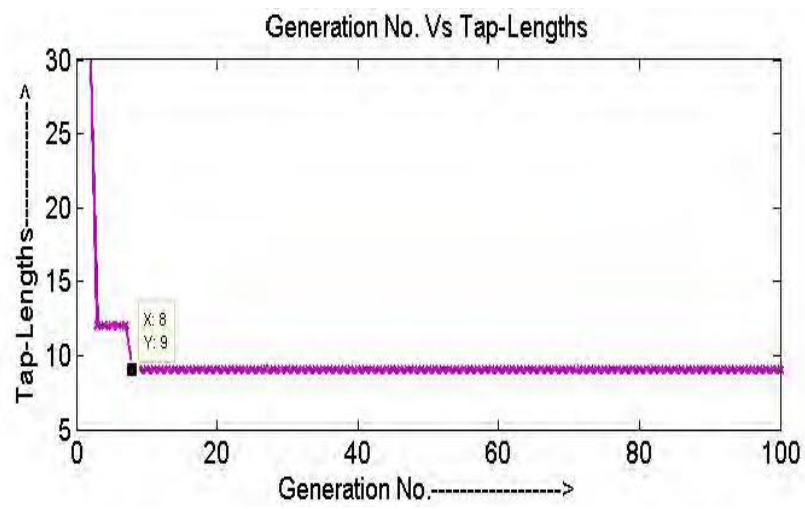

Fig-2: Tap-Length learning curve generations.
Tap-lengths having least MSE from first generation to 100 th generation are plotted in Fig.2. It shows that after 8th generation tap-length gets constant value i.e. 9 which is exactly the same as that of the unknown system. It implies that the realized system with the proposed adaptation technique can work well in changing environments. The simulation result also implies that there is the most probability of getting optimal tap-length very fast that is convergence is good in terms of tap-length adaptation.

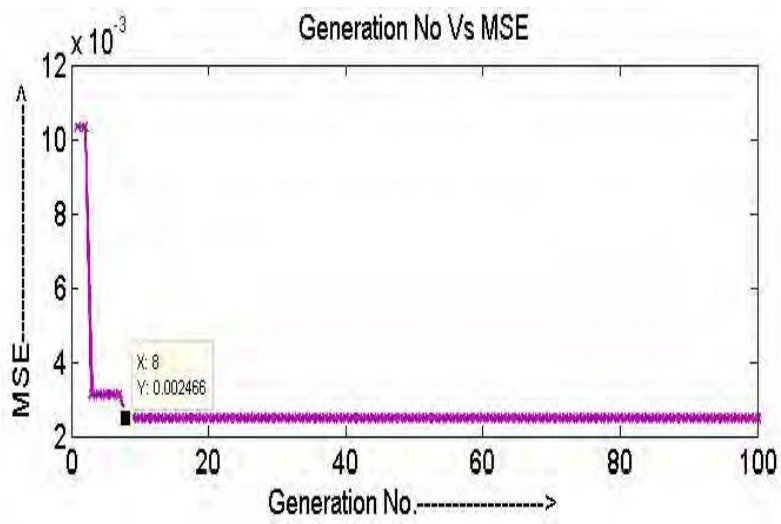

Fig-3: MSE learning curve with corresponding generations

Mean of MSE taking from last five hundred samples for every individual tap-length are calculated and least MSE of every generation has plotted here in Fig.3. After 8th generation it gets least MSE i.e. 0.002466 and Thereafter it does not change until system is changed.Fig. 4 shows the MSE learning curve with corresponding tap-length. MSE is less when tap-length is nine and elsewhere it increases. So, here optimum tap-length is 9 .

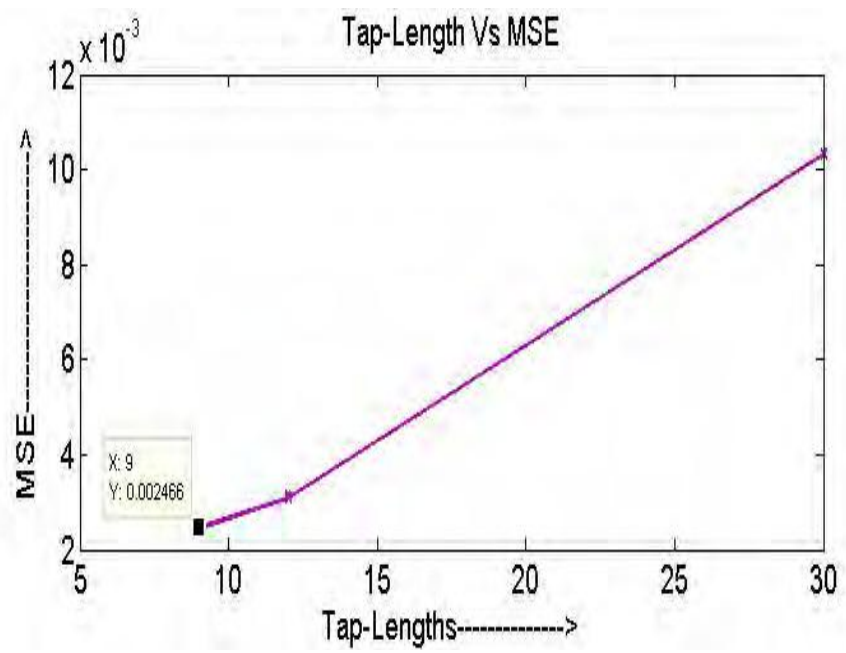

Fig-4: MSE learning curve with respect to corresponding TapLength. 


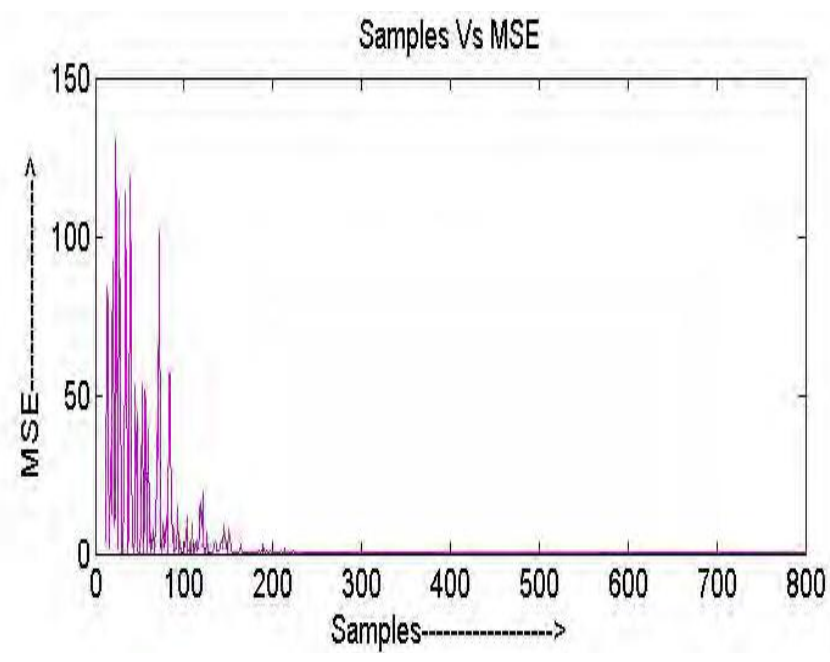

Fig-5: MSE learning curve with respect to corresponding samples using LMS when $\mu=0.005$.

Fig. 5 shows the MSE learning curve with respect to input samples when tap-length is 9 (optimum). Here tap weights are updated using LMS with $\mu=0.005$. This figure indicates that error curve converges very fast by updating tap-weight vector using LMS and it shows that MSEs are significantly less after 160 samples.

\section{CONCLUSIONS}

This technique of adaptation gives better performance over only LMS based optimization as one of the important parameter tap-length is updated using PSO while another parameter weight-vector is updated using LMS. If both parameters are updated using LMS, it will increase adaptive noise, initialization problem and slow down the convergence rate. Besides PSO style variable tap-length can be applied to different engineering applications such as smart antenna, channel equalizer, echo canceller etc. because it is easy to understand, has low adaptation noise and simple fitness function. These benefits may make it capable to lead recent research areas (adaptive) like optimization, optimal tap-length determination, control system design etc. In the LMS it requires large numbers of iterations to find optimal tap-length but using the PSO it is easy to get the same very fast. So, it reduces the computational complexity which is desirable to be minimized in time varying environment as training phase is executed time to time. Also in LMS, tap-length learning curve may gets stuck in a local minima so optimum tap-length determination is not so easy. The PSO introduces the principle of evolution that use "Good solution survives while bad one dies" and does not depend on derivatives and based on natural selection. Also, the PSO with small solution size and high mutation rates give best solution. Hence proposed PSO style algorithm for tap-length adaptation can take attention to determine optimal solution in adaptive system.

\section{ACKNOWLEDGEMENTS}

The authors would thanks the Jyothy Institute of Tech, Management, Principal of JIT for there support and reviewers for their help in improving the document.

\section{REFERENCES}

[1]. Solmaz, C.O.; Oruc, O.; Kayran, A.H.; “ Optimal stepsize LMS equalizer algorithm ".Signal Processing and Communications Applications (SIU), 2011 IEEE . April 2011,page no. 853 - 856 .

[2]. Wee-Peng Ang Farhang-Boroujeny, B," A new class of gradient adaptive step size LMS algorithm" "Signal Processing, IEEE Transactions on,2001, Volume: U page(s): $805-810$.

[3]. Krstajic, B. Stankovic, L.J. Uskokovic, Z. "an approach to variable step size LMS algorithm". : Electronics Letters , Aug 2002 ,Volume: 38 Issue: 16,On page(s): 927 - 928

[4]. Saul B. Gelfand, Yongbin Wei, James V. Krogmeier, ," The Stability of Variable Step-Size LMS Algorithms" IEEE TRANSACTIONS ON SIGNAL PROCESSING, VOL. 47, NO. 12, December 1999

[5]. R. Kwong and E. W. Johnston, "A variable step size LMS algorithm,” IEEE Trans. Signal Processing, vol. 40, pp. 1633 1642, July 1992.

[6]. [11] V. J. Mathews and Z. Xie, "A stochastic gradient adaptive filter with gradient adaptive step size," IEEE Trans. Signal Processing, vol. 41,pp. 2075-2087, June 1993.

[7]. Tao Dai; Shahrrava, B.," Variable step-size NLMS and affine projection algorithms with variable smoothing factor "Circuits and Systems, 2005. 48th Midwest Symposium on , Aug. 2005 , page(s): 1530 - 1532 Vol. 2. 